\title{
Biodiversité et diversité culturelle : trajectoire d'une analogie (2001-2010)
}

Biodiversity and cultural diversity: trajectory of an analogy (2001-2010)

\section{Irit Sholomon-Kornblit}

\section{(2) OpenEdition \\ 1 Journals}

Édition électronique

URL : http://journals.openedition.org/aad/2711

DOI : 10.4000/aad.2711

ISSN : 1565-8961

\section{Éditeur}

Université de Tel-Aviv

\section{Référence électronique}

Irit Sholomon-Kornblit, « Biodiversité et diversité culturelle : trajectoire d'une analogie (2001-2010)», Argumentation et Analyse du Discours [En ligne], 21 | 2018, mis en ligne le 15 octobre 2018, consulté le 23 septembre 2019. URL : http://journals.openedition.org/aad/2711 ; DOI : 10.4000/aad.2711

Ce document a été généré automatiquement le 23 septembre 2019

\section{(c) $(1) \odot$}

Argumentation \& analyse du discours est mis à disposition selon les termes de la licence Creative Commons Attribution - Pas d'Utilisation Commerciale - Pas de Modification 4.0 International. 


\section{Biodiversité et diversité culturelle : trajectoire d'une analogie (2001-2010)}

Biodiversity and cultural diversity: trajectory of an analogy (2001-2010)

Irit Sholomon-Kornblit

\section{Introduction}

1 L'article premier de la Déclaration universelle de l'UNESCO pour la diversité culturelle (désormais : DUDC) déclare que « la diversité culturelle est, pour le genre humain, aussi nécessaire qu'est la biodiversité dans l'ordre du vivant " (UNESCO 2002, art. $1^{\text {er) }}$. À partir de cette analogie comparative, l'association entre diversité biologique et culturelle se fait de plus en plus serrée dans le discours de l'UNESCO, allant jusqu'à sa fusion, sous la forme de "biodiversité culturelle », dans la Convention de la biodiversité culturelle en 2010 (UNESCO 2010b).

On peut qualifier ce néologisme métaphorique d'« analogie condensée » (Perelman et Olbrechts-Tyteca 1988 : 535). Nous proposons ici de retracer le parcours qui y a mené en reconstruisant l'évolution des diverses formes d'analogies associant diversité biologique et culturelle dans le discours de l'UNESCO. Ce discours comprend, outre la DUDC, deux Rapports Mondiaux de la Culture de l'UNESCO (2000, 2010a); deux brochures autour du Festival de la diversité culturelle et autour d'une exposition en l'honneur de la DUDC (UNESCO 2002, 2010c), la Convention de la biodiversité (ONU 1992) et le compte rendu de la Conférence pour la diversité biologique et culturelle (UNESCO 2010b). Le corpus comprend en outre le métadiscours scientifique publié par l'UNESCO (Appadurai 2002, Lévi-Strauss 2007, Mattelart 2007, Bandarín 2007).

3 Au sein d'une approche combinant analyse du discours et argumentation, nous nous intéressons également aux visées argumentatives de l'UNESCO à travers ce mouvement analogique. Celles-ci sont multiples : en premier lieu, au moment de l'apparition de la 
diversité culturelle à l'UNESCO, la biodiversité est déjà un concept bien établi sur la scène internationale, consacré depuis 1992 dans la Convention pour la biodiversité (ONU 1992). On sait que l'analogie est un outil heuristique important grâce à sa faculté de faire saisir l'inconnu au moyen du connu à travers des similitudes (Perelman et Olbrechts-Tyteca 1988 : 531). La comparaison de la diversité culturelle à la diversité biologique permet donc de faire accepter la première plus facilement par le public : non seulement du point de vue cognitif, mais aussi grâce à l'autorité d'antécédent dont jouit la biodiversité. Populairement associé au discours scientifique ${ }^{1}$, le concept de biodiversité jouit, de plus, de l'autorité procurée par la science (Angenot 2013: 122-123). Enfin, la biodiversité bénéficie d'un statut de " bien-penser» et de «politiquement correct»: c'est une valeur pleinement reconnue par la communauté internationale. Liée au discours du développement durable et de l'écologie, elle est de «bon ton »; elle permet d'associer à la diversité culturelle des valeurs positives et de l'intégrer dans la doxa.

4 Nous commençons par un aperçu théorique où nous définissons les concepts-clés et nos outils méthodologiques. Nous passons ensuite à l'analyse d'extraits représentant les différentes étapes de cette évolution, allant de différents types d'analogies à une étape intermédiaire, en terminant par le "dépassement», ou la pleine réalisation de l'analogie dans la formule de « biodiversité culturelle $»^{2}$.

\section{Définitions}

\section{Diversité culturelle et biodiversité}

5 La diversité culturelle n'est pas définie dans la DUDC; elle ne le sera que quatre ans plus tard, dans la Convention sur la protection et la promotion de la diversité des expressions culturelles, qui la définit comme « la multiplicité des formes par lesquelles les cultures des groupes et des sociétés trouvent leur expression» (UNESCO 2005, article 4.1). Ce manque de définition lors de la Déclaration censée la consacrer pourrait bien expliquer le recours à l'analogie qui offre un concept proche déjà bien ancré dans le discours international ; il représenterait un effort de mieux circonscrire un concept encore mal établi.

Inversement, la notion de biodiversité apparaît déjà sur la scène scientifique américaine dans les années 1980, dans la continuation du mouvement environnementaliste global de conservation ${ }^{3}$. L'ONU l'adopte officiellement dans son discours lors de la Convention des Nations Unies sur la biodiversité de 1992, qui la définit comme la "variabilité des organismes vivants de toute origine [...] cela comprend la diversité au sein des espèces et entre espèces ainsi que celle des écosystèmes » (ONU 1992:3).

\section{Analogies}

7 En rhétorique, l'analogie est une "similitude de structures» (Perelman et OlbrechtsTyteca $1988: 500$ ), dont la formule générale serait $A$ est à $B$ ce que $C$ est à $D$, lorsque $A$ et $B$ constituent le thème de l'analogie et $C$ et $D$ en constituent le phore (ibid. : 500-501). Le phore est mieux connu que le thème, et sert à en éclairer la structure ou à en établir la valeur (ibid.: 501, 512-513), permettant ainsi de le situer dans un cadre conceptuel 
(ibid. : 517). Les arguments par l'analogie établissent un parallèle entre ce dont on est en train de parler (le thème) et un autre objet ou une autre situation (le phore) sur la base d'une relation de ressemblance ponctuelle et partielle entre les deux, pour faire passer une propriété ou une relation admise à propos du phore sur le thème, qui appartient à un domaine sémantique différent.

Bien que les auteurs du Traité de l'argumentation excluent la comparaison de cette catégorie, la considérant comme un "argument quasi-logique ", nous avons choisi de l'inclure, avec Marc Bonhomme et Christian Plantin, sous le chapeau des figures analogiques, ou plus largement des procédés rhétoriques de ressemblance. Pour Bonhomme (1998: 66), la comparaison «établi[ $t]$ une analogie entre des termes appartenant à des domaines notionnels [...] différents en vertu d'une communauté de sens» (je souligne). Plantin (2011: 114) inclut l'analogie par comparaison dans la catégorie des analogies relationnelles ${ }^{4}$. Ce qui importe donc, à nos yeux, du point de vue heuristique, rhétorique et argumentatif, est l'analogie sous-tendant la comparaison et moins le fait que celle-ci soit, oui ou non, marquée linguistiquement (Hilgert 2016 : 80); on peut ainsi concevoir la comparaison (avec la métaphore) comme une ellipse du procédé analogique (ibid.).

Roselyne Koren (2016), suite à sa constatation d'une "porosité des limites entre ces diverses figures» et «le fait qu'elles sont souvent présentées comme interchangeables» ( $\$ 28)$, propose la notion d'«air de famille» que ces figures partagent autour de la « ressemblance » (\$ 26). Chacun de ces arguments contribue en effet à sa manière, en fonction de sa structure spécifique, à l'élucidation (toujours partielle et approximative) de cette ressemblance « qui joue un rôle cognitif et évaluatif essentiel dans la vie politique et sociale de l'individu et de la collectivité à laquelle il appartient " (ibid.). Comme, dans notre corpus, ces différents types de figures de ressemblance coexistent et s'interchangent souvent autour du même rapprochement conceptuel, nous proposons de voir dans l'amalgame entre ces figures l'« hésitation de l'homme incertain qui use du langage et des pratiques discursives et argumentatives afin d'explorer et de justifier ce qui vaut et fait sens pour lui dans un contexte sociohistorique en perpétuelle évolution » (\$28). Nous appliquerons donc à notre corpus cette perspective de « ressemblance de famille » qui témoigne des changements dans les perceptions qu'a la société de la relation et la catégorisation de certains concepts flous, abstraits, complexes et mouvants.

\section{Trajectoire de l'analogie effectuée entre diversité biologique et culturelle}

10 Nous tracerons le parcours analogique du rapprochement entre diversité culturelle et biologique en usant de la tripartition de Plantin. Nous commençons par l'analogie relationnelle (Plantin $2011: 317$ ), qui est une analogie entre deux relations mettant en jeu quatre termes, sous la forme d'A est à $B$ ce que $C$ est à $D$. Ce type d'analogie correspond, dans la rhétorique classique et nouvelle, au modèle-type de l'analogie (Perelman et Olbrechts-Tyteca 1988: 500). Nous passons ensuite à des exemples de l'analogie catégorielle, où deux éléments analogues sont intégrés à une même catégorie. Ce type d'analogie correspond, en rhétorique, aux arguments quasi-logiques telles la règle de justice, l'identification ou l'inclusion de la partie dans le tout (294, 282, 311). L'analogie catégorielle constitue déjà, d'après nous, une étape vers le dépassement 
de l'analogie, dans la mesure où les deux éléments y sont déjà considérés comme moins hétérogènes (528-530). La dernière catégorie d'analogie que cite Plantin $(2011: 117)$ est l'analogie structurelle ou l'isomorphisme, qui combine les deux premiers types d'analogie: il s'agit d'une analogie entre deux systèmes complexes partageant une même structure. Après l'association graduellement plus intense entre les deux types de diversité, on montre comment les deux fusionnent dans le néologisme de «biodiversité culturelle ».

\section{L'analogie relationnelle}

11 L'article premier de la DUDC déclare que «la diversité culturelle est, pour le genre humain, aussi nécessaire qu'est la biodiversité dans l'ordre du vivant " (UNESCO 2002). L'analogie est ici marquée par la présence des quatre éléments de la formule: la diversité culturelle (A), le genre humain (B), la biodiversité (C) et l'ordre du vivant (D). Le degré de nécessité (" aussi nécessaire que ») est «l'air de famille », la ressemblance ponctuelle et partielle effectuée entre les deux catégories du culturel et du biologique. On peut la schématiser ainsi :

\begin{tabular}{|l|l|}
\hline Thème & Phore \\
\hline A. La diversité culturelle & B. La biodiversité \\
\hline C. Le genre humain & D. L'ordre du vivant \\
\hline
\end{tabular}

Table 1 : Analogie relationnelle : «la diversité culturelle est, pour le genre humain, aussi nécessaire qu'est la biodiversité dans l'ordre du vivant » (UNESCO 2002, art. 1er)

12 Selon la formule analogique d'A est à $B$ ce que $C$ et à $D$, la diversité culturelle (A) est nécessaire au genre humain $(B)$ au même titre que la biodiversité (C) est nécessaire à l'équilibre naturel (D). C'est là une analogie par comparaison (Plantin 2011: 114): marquée grammaticalement par "aussi nécessaire que ", la relation entre la diversité culturelle et le genre humain est associée à celle qui régit biodiversité et Nature. La communauté de sens établie par l'analogie-comparaison est le degré de nécessité.

13 À travers le rapprochement entre les relations des éléments du phore avec ceux du thème, l'analogie a pour but ultime de rapprocher le thème du phore (Perelman \& Olbrechts-Tyteca $1988: 508,528-530$ ). Durant le processus d'analogie, une propriété ou une relation attribuée au domaine-source (correspondant au phore) est transférée ou projetée sur le domaine-cible (correspondant au thème). Comme le domaine-source est mieux connu et établi que le domaine-cible, le premier est projeté sur le dernier, permettant de le cadrer et aidant à l'interpréter (512-517). Le domaine-source fonctionne donc comme une « ressource » qui sert à « résoudre » ce que Plantin (2011 : 118) appelle le "domaine-problème ». Ici le thème, plus nouveau, de la diversité culturelle, est argumenté à l'aide du phore qui est déjà accepté dans la société : l'importance de la biodiversité dans l'équilibre mondial naturel. Le savoir et la doxa partagés regardant la biodiversité, le rôle que celle-ci joue dans la santé et l'écologie, les valeurs qui lui sont associées, son statut de légitimité épistémologique et politique, sa stabilité normative et linguistique en tant que concept déjà établi dans la société et 
dans la communauté linguistique - tous ces traits sont projetés, lors du procédé d'analogie, sur le domaine-cible de « diversité culturelle » (cf. Plantin $2016: 387$ ).

Un autre exemple de l'analogie relationnelle entre diversité biologique et culturelle apparaît déjà un an plus tôt, dans le Rapport Mondial de la Culture de 2000 : «la perte de la diversité culturelle se déroule en interaction avec la perte de la diversité biologique » (Arizpe et al. $2000: 26$, je souligne). La nature de cette interaction n'est pas spécifiée : est-ce une relation de coexistence, où l'on assiste à la perte simultanée des deux types de diversité de manière indépendante, ou une relation de causalité qui les lie ? Ce n'est pas clair. Si l'analogie fondée sur la comparaison confronte deux domaines distincts sans les confondre (Plantin $2016: 388$ ), une relation de causalité entre thème et phore représenterait déjà un dépassement de l'analogie (Perelman et Olbrechts-Tyteca 1988 : 529-530).

15 Un graphique figurant dans ce Rapport (UNESCO $2000: 26$ ) illustre cette analogie en juxtaposant la biodiversité et la diversité linguistique de certains pays, montrant une corrélation entre les deux types de diversité, mais toujours sans spécifier la nature de cette corrélation. Elle vérifie combien de langues (pour la plupart autochtones) existent dans les vingt pays à la plus grande biodiversité dans le monde : dans douze pays, il y a plus de 50 langues parlées. Ce graphique associe un type spécifique de diversité à la biodiversité : la diversité linguistique, ici intimement liée à la diversité de cultures traditionnelles et orales. Ce rapprochement, qui se concentre sur la diversité culturelle des peuples autochtones, constitue un pas, on le verra, vers la fusion des deux types de diversité, biologique et culturelle.

Le second Rapport Mondial sur la Culture de l'UNESCO (2010a) construit une analogie associant les diversités biologique et culturelle relativement à la menace semblable de disparition qui pèse sur elles, et qui est attribuée aux processus de modernisation et de mondialisation: «Les scientifiques [...] esquiss[e]nt un parallèle entre érosion de la biodiversité et disparition des modes de vie traditionnels du fait de la raréfaction des ressources et de la généralisation des modes de vie modernes » (1).

\begin{tabular}{|l|l|l|}
\hline & Thème & Phore \\
\hline Effet & A. la disparition des modes de vie traditionnels & C. l'érosion de la biodiversité \\
\hline Cause & B. la généralisation des modes de vie modernes & D. la raréfaction des ressources \\
\hline
\end{tabular}

Table 2 : Analogie relationnelle : « Les scientifiques esquissent un parallèle... » (UNESCO 2010a : 1)

Selon cette analogie, qui se présente comme une analogie relationnelle de cause, de même que la perte de biodiversité mène inévitablement à la raréfaction des ressources (domaine-source ou phore), ainsi la perte de diversité culturelle, ici réduite aux "modes de vie traditionnels", entrainerait la "généralisation de modes de vie modernes » (domaine-cible ou thème). La "diversité culturelle» désigne ici seulement les cultures traditionnelles, en danger d'extinction : l'analogie avec la biodiversité et le monde du vivant se fonde donc sur une menace existentielle imminente. On note pourtant la relation quelque peu tautologique sous-jacente à la présentation qui pose une relation de cause à effet : la «disparition des modes de vie traditionnels » est plus ou moins synonyme de la "généralisation des modes de vie modernes »; « l'érosion de 
la biodiversité » est synonyme de la « raréfaction des ressources ». Il s'agit d'une fallacy d'expression, qui présente comme une relation de cause à effet ( du fait de...») des phénomènes synonymes afin de justifier la relation analogique entre perte de diversité culturelle et biologique. Or cet argumentaire est présenté comme venant de la bouche des "scientifiques ", mimant une pensée rationnelle et une source d'autorité.

L'analogie effectuée entre ces deux relations charge implicitement la perte de diversité culturelle de valeur axiologique négative : comme la perte de biodiversité (apparaissant ici sous la métaphore géologique de l'érosion) est un phénomène négatif menant à la raréfaction des ressources, il s'ensuit, par la force de la forme analogique, que la disparition de modes de vie traditionnels est un phénomène tout aussi négatif (et dangereux pour la survie de l'humanité, selon tout l'interdiscours vu jusqu'à présent). Cet exemple montre comment l'analogie peut aussi transférer des valeurs à côté des contenus sémantiques. Ces jugements de valeur ont été introduits par l'analogie comparative en allant du domaine de la géologie («érosion») qualifiant ici la biodiversité qui est un phénomène biologique, au domaine du culturel (les modes de vie traditionnels versus les modes de vie modernes), pour terminer par le domaine axiologique qui se manifeste à travers les substantifs d'« érosion ", de " raréfaction » et de "disparition", impliquant tous une perte et donc chargés de valeur affective négative. Tout cela est légitimé par l'autorité des scientifiques qui utilisent des termes comme « érosion ", « raréfaction des ressources », ou « disparation de modes de vie ».

\section{L'analogie catégorielle}

19 Nous verrons ici des exemples d'analogie de scientifiques employés comme experts par l'UNESCO : le premier, Arjun Appadurai, est un chercheur en cultural studies sollicité par l'UNESCO pour un article apparaissant dans une publication en l'honneur de la DUDC. Le second est Claude Lévi-Strauss, ethnologue français de grande renommée, associé de longue date avec l'UNESCO. L'UNESCO, elle-même autorité internationale reconnue, recourt fréquemment à l'autorité d'« experts » (Cussó et Gobin 2008) : les comités de rédaction des Déclarations et des Conventions en appellent aux services d'économistes, d'anthropologues, de sociologues et de juristes. Plus qu'une crédibilité objective, il s'agirait, selon Angenot (2013: 99), d'une crédibilité principalement liée au seul prestige de l'expert. Le recours à l'autorité d'experts ou de savants contribue à la construction de l'ethos de l'UNESCO comme une institution crédible, honnête, raisonnable, dotée de bonne volonté et de prudence. L'institution se montre « en quête du vrai, du juste et du bien [s'en remettant] au mieux informé, plus compétent ou plus sage et s'en fait le porte-parole » (ibid.: 48-50), gommant tout aspect idéologique du discours onusien sur la diversité culturelle. Tout en étant elle-même créatrice de doxa, en vertu de son statut d'institution mondialement reconnue jouissant d'un pouvoir symbolique, l'UNESCO prétend ainsi être le porte-parole de la volonté générale.

Appadurai déclare: «Il est aujourd'hui largement reconnu qu'une homologie existe entre la biodiversité et la diversité culturelle» (Appadurai 2002: 14). L'autorité ne provient plus des «scientifiques": elle dérive tout simplement du pronom personnel neutre «il»(«il est largement reconnu»), qui renvoie à la doxa. L'analogie est ici marquée par le substantif « homologie ». L'auteur explique plus loin en quoi consiste, à ses yeux, cette homologie :

Dans les deux cas [diversité culturelle et biodiversité], un attachement aveugle et monothéiste aux principes du marché entraîne la marginalisation des valeurs 
transhistoriques. La diversité culturelle et la biodiversité sont chacune des valeurs

du et pour le long terme (ibid. : 15).

21 Appadurai établit ici une analogie catégorielle : les deux types de diversité sont des "valeurs transhistoriques", qui sont menacées par les forces de la mondialisation, décrite ici à l'aide d'un lexique religieux (« un attachement aveugle et monothéiste »). Ce choix de langage insinue qu'il s'agit ici d'un discours idéologique, contrairement au discours "scientifique " qu'on attend de l'expert. Cette idéologie du marché est axiologiquement chargée de façon négative: elle entraîne la marginalisation de certaines valeurs ${ }^{5}$. Diversité biologique et culturelle sont représentées comme un contre-discours appartenant à une catégorie unique : celle des «valeurs du et pour le long terme ». Les deux types de diversité sont donc, contrairement à la mondialisation, positivement chargés du point de vue axiologique, suite à une mise en valeur de la dimension éthique de la diversité. Comme le souligne le géographe Laurent Simon (2006 : 461), « la notion [de biodiversité] est chargée de normes de valeur : elle est ce qui est naturel, ce qui est vulnérable, ce qui enfin est bon pour l'homme et la survie de l'humanité ». Toutes ces normes de valeurs sont projetées sur la diversité culturelle lors des analogies effectuées entre elles.

\section{L'analogie structurelle ou isomorphisme}

Lévi-Strauss, un des ethnologues les plus étroitement associés à l'UNESCO, élabore lui aussi l'homologie existant à ses yeux entre diversité culturelle et biologique lorsqu'il dit, en tant qu'invité d'honneur au Congrès à l'occasion du cinquantième anniversaire de l'UNESCO, que celle-ci « a toujours reconnu qu'une correspondance existe entre la diversité culturelle et la biodiversité » (Lévi-Strauss 2007 : 35).

Il y a ici une circularité lorsque Lévi-Strauss, qui représente une figure majeure d'autorité pour l'UNESCO, cite à son tour cette institution comme figure d'autorité. De plus, la dimension temporelle introduite ici avec «a toujours reconnu que » ajoute encore de la légitimité par la voie de l'autorité de l'histoire : il en a toujours été ainsi, il s'agit d'une des pierres angulaires des valeurs de l'UNESCO. Lévi-Strauss élabore par la suite l'analogie qu'il indique ici par la voie du substantif « correspondance »:

les conditions [pour distinguer entre une culture de ses voisines] sont grosso modo les mêmes que celles qui favorisent la différenciation biologique : isolement relatif pendant un temps prolongé, échanges limités, qu'ils soient d'ordre culturel ou génétique. Au degré près, les barrières culturelles sont de même nature que les barrières biologiques. Celles-ci les préfigurent d'une manière d'autant plus véridique que toutes les cultures impriment leur marque au corps par des styles de costume, de coiffure et de parure, par des mutilations corporelles et par des comportements gestuels, elles miment des différences comparables à celles qu'on reconnaît entre les variétés au sein d'une même espèce (ibid.).

Lévi-Strauss établit ici un isomorphisme, une analogie structurelle, entre biodiversité et diversité culturelle. Nous rappelons qu'une analogie structurelle est une analogie entre deux systèmes complexes partageant une même structure. Combinant l'analogie catégorielle et l'analogie de relation, ce type d'analogie repose sur un nombre indéfini $a$ priori d'objets et de relations à l'intérieur de cette structure (Plantin 2011 : 117). La reconstruction de l'analogie établie ici donne ceci :

\begin{tabular}{|l|l}
\hline Biodiversité & Diversité Culturelle \\
\hline
\end{tabular} 


\begin{tabular}{|l|l|}
\hline \multicolumn{2}{|l|}{ Isolement relatif pendant une période prolongée } \\
\hline Échanges limités d'ordre génétique & Échanges limités d'ordre culturel \\
\hline $\begin{array}{l}\text { Différences dans le phénotype entre les } \\
\text { variétés au sein d'une même espèce }\end{array}$ & $\begin{array}{l}\text { Style de coiffure, de costume et de parure, } \\
\text { mutilations corporelles, comportements gestuels }\end{array}$ \\
\hline
\end{tabular}

Table 3 : Isomorphisme entre diversité biologique et culturelle (Lévi-Strauss 2007 : 35) stratégies de différentiation: si, dans le cas de la biodiversité, il s'agit de différenciations marquées par le géno- et phénotype, dans le cas de la diversité culturelle, il s'agit d'une différenciation marquée par les coutumes, par les parures, en d'autres termes, par la culture. Les relations mises en analogie dans cet isomorphisme sont celles entre les espèces ou les cultures, selon les domaines respectifs, concernant les barrières que celles-ci construisent pour mieux se différencier. Ces pratiques de différenciation sont, en outre, présentées comme essentielles à la survie et à l'adaptation à l'environnement ; les hommes ne feraient que continuer une tradition de longue date, ayant ses racines dans les processus naturels et donc nécessaires. De même que, dans le cas de la Nature, l'isolement relatif pendant une période prolongée mène à un échange génétique limité, chez l'Homme cet isolement mène à des échanges limités d'ordre culturel ; de même que, dans la Nature, ces échanges génétiques limités mènent à une différenciation du phénotype de cette espèce par rapport à d'autres espèces, chez l'Homme, les échanges culturels limités mènent à des style de coiffure, de costume, de gestuelle et de mutilations corporelles spécifiques à cette espèce.

isomorphisme est marqué à plusieurs reprises dans le texte, indiquant le caractère didactique des propos de Lévi-Strauss, qui cherche à légitimer et à expliquer son point de vue : les conditions de distinction de B «sont grosso modo les mêmes que » celles de A (isolement et échanges limités) ; les barrières de $B$ sont, " au degré près ", " de même nature que » celles d'A, qui « préfigurent » celles de B, c'est-à-dire servent de modèle à celles de B, qui « miment » des différences « comparables à » celles de A. Lévi-Strauss parle ici en ethnologue, en scientifique, se limitant à citer des «observables » sans jugement de valeur aucune. Il poursuit son argument ainsi :

Diversité culturelle et diversité biologique ne sont donc pas seulement des phénomènes du même type. Elles sont organiquement liées, et nous nous apercevons chaque jour davantage qu'à l'échelle humaine, le problème de la diversité culturelle reflète un problème beaucoup plus vaste et dont la solution est encore plus urgente, celui des rapports entre l'homme et les autres espèces vivantes, et qu'il ne servirait à rien de prétendre le résoudre sur le premier plan si l'on ne s'attaquait pas aussi à lui sur l'autre, tant il est vrai que le respect que nous souhaitons obtenir de chaque homme envers les cultures différentes de la sienne n'est qu'un cas particulier du respect qu'il devrait ressentir pour toutes les formes de la vie (ibid., je souligne).

Cette rupture dans le discours de Lévi-Strauss marque l'étape suivante de notre parcours analytique: on passe de la comparaison entre les deux catégories de biodiversité et de diversité culturelle, qui présuppose toujours la distinction entre ces deux catégories afin de pouvoir effectuer une analogie entre elles (entre des " phénomènes du même type »), à un stade où l'un (la diversité culturelle) est considéré comme faisant partie de l'autre (la biodiversité). Dans la mesure où chaque homme se doit d'éprouver du respect pour "les cultures différentes de la sienne », la diversité 
culturelle devient une valeur et non pas un observable ethnologique. En d'autres termes, elle n'est pas seulement un "cas particulier » de la biodiversité, mais une valeur à laquelle chacun doit apporter son tribut : « le respect que [l'homme] devrait ressentir pour toutes les formes de vie ». Les deux sont présentées comme étant " organiquement liées "; la diversité culturelle est une partie appartenant à un Tout qui la surplombe, « un problème beaucoup plus vaste dont la solution est encore plus urgente ». Ce problème est celui des " rapports entre l'homme et les autres espèces vivantes ». Dès lors que l'homme est recatégorisé parmi les " autres espèces vivantes ", le problème de la diversité culturelle est dépolitisé ; il est présenté comme appartenant au domaine de l'écologie.

Dans la partie suivante, nous analyserons des extraits où diversité culturelle et biologique ne sont plus des catégories séparées mais apparaissent comme « organiquement liées".

\section{Dépassement de l'analogie : la biodiversité et la diversité culturelle « organiquement liées »}

Si l'on rappelle la définition de la biodiversité comme la « variabilité des organismes vivants de toute origine [...]; cela comprend la diversité au sein des espèces et entre espèces ainsi que celle des écosystèmes » (ONU 1992: 3), on voit aisément comment l'homme peut être inclus dans la biodiversité. Il est bien un « organisme vivant » et il fait partie intégrante, depuis le mouvement écologiste, des écosystèmes ${ }^{6}$. Le statut "séparé » des domaines rapprochés par l'analogie est donc un statut ambigu et mouvant, ce qui mène à un mélange des domaines du biologique et du culturel dans le discours sur la diversité. Ainsi, une brochure du Festival de la diversité culturelle de 2010 parle de celle-ci en termes biologiques lorsqu'elle la qualifie de " condition naturelle de l'espèce humaine " (Rivière 2010). Les deux syntagmes de "condition naturelle » et d'" espèce humaine » appartiennent clairement au registre biologique et scientifique. Ce phénomène n'est pas nouveau dans le discours onusien: le Rapport mondial de la Culture de 2000 qualifie déjà la culture de «mutation génétique » :

Bien que les êtres humains soient une espèce animale, c'est la mutation génétique leur ayant donné cette capacité d'apprendre et de transmettre le savoir d'une génération à la suivante qui explique le succès historique de l'expansion des populations humaines dans le monde entier. Malheureusement, cette capacité conduit à la destruction de la biodiversité sur la planète avec des conséquences imprévisibles (Arizpe et al. $2000: 27$ ).

Ce paragraphe met en scène une humanité qui fait partie intégrale de la Nature, son écosystème: l'humanité est qualifiée $\mathrm{d}^{\prime}$ ' espèce animale» qui, grâce à une «mutation génétique » a connu un succès formidable du point de vue historique. Ces syntagmes, tout comme celle des "populations humaines ", proviennent, ici aussi, du registre scientifique biologique. Or, cette espèce parmi d'autres a conduit à la « destruction de la biodiversité sur la planète ». Non seulement parle-t-on de la culture en termes biologiques dans un Rapport sur la Culture, mais encore la capacité humaine de générer de la culture est immédiatement liée de façon causale ("conduit à ») à la destruction de la biodiversité et donc présentée dans l'optique, très spécifique, de l'impact de cet animal cultivé sur son environnement. Sous-entendant que l'homme devrait connaître sa place et ne pas se percevoir comme supérieur aux autres espèces 
peuplant la planète, "l'expansion des populations humaines dans le monde entier " décrit le statut de l'homme en termes d'impérialisme et même de colonialisme. Les auteures poursuivent: "alors que la biodiversité a été estimée à plus de 30 millions d'espèces, les humains depuis la disparition de l'homme de Neandertal et du pithécanthrope, ne sont plus qu'une unique espèce Homo, avec aujourd'hui plusieurs milliers de modes de vie différents » (ibid.).

Le connecteur d'opposition "alors que » indique une mise en relation de contradiction entre la biodiversité "estimée à plus de 30 millions d'espèces ", et les humains qui aujourd'hui «ne sont plus qu'une unique espèce Homo ». Si jadis, l'humanité comptait une diversité biologique avec le Neandertal et le pithécanthrope, celle-ci a été perdue et remplacée par une diversité d'ordre culturel: les "milliers de modes de vies différents ». À travers la relation de contradiction entre biodiversité et diversité culturelle construite ici, les auteures semblent vouloir dire la chose suivante: non seulement on a-t-on perdu la diversité biologique qu'il y avait jadis entre les hominoïdes, mais on risque également de perdre cet autre type de diversité qu'est la diversité culturelle. La biodiversité sert ici de modèle à la diversité culturelle de l'homme : celui-ci doit chérir et protéger sa diversité, comme il doit chérir et protéger la biodiversité.

La diversité culturelle est donc présentée ici comme un type supplémentaire de biodiversité, comme une étape évolutive de cette dernière chez l'homme : les « milliers de modes de vies différents " sont mis en relation d'équivalence avec les "plus de 30 millions d'espèces ». Un rapprochement très significatif s'opère ainsi entre deux catégories en chemin vers une seule supercatégorie. À travers ce rapprochement, dans la cadre du processus déjà entamé dans les passages commentés plus haut, la catégorie de biodiversité semble avoir assimilé celle de diversité culturelle, à travers la technique rhétorique de l'inclusion de la partie dans le tout où « le tout englobe la partie et, par conséquent, est plus important qu'elle» (Perelman et Olbrechts-Tyteca 1988: 311). Dans ce procédé de rapprochement, l'homme est « une unique espèce Homo » parmi les « 30 millions d'espèces ».

Cette ligne d'argumentation contredit un passage antérieur du même texte, qui retrace la diversité culturelle à " l'aube de la vie humaine », où la "différence entre hominidés et Homo sapiens était culturelle» (Arizpe 2000: 26). Ce phénomène confirme le sentiment que les deux champs de biodiversité et de diversité culturelle deviennent de plus en plus entremêlés du point de vue sémantique. Ce flou des frontières sémantiques peut être très efficace du point de vue argumentatif, permettant de choisir la catégorisation adéquate d'après le type d'argumentation mobilisé. Ici, le fait de biologiser la diversité culturelle permet de naturaliser cette dernière ; faisant partie de l'ordre naturel, existant dès "l'aube de la vie humaine", elle devient difficilement réfutable ; son importance s'en trouve donc encore davantage légitimée.

\section{Fusion en la « biodiversité culturelle »}

Le thème et le phore de l'analogie entre la diversité culturelle et la biodiversité finissent par se fondre en une seule catégorie: la "biodiversité culturelle». Cette formule condensée, dont l'adjectif est 'valisé' dans la version anglaise originale (biocultural diversity), est définie pour la première fois dans un document de travail de la Conférence internationale sur la diversité biologique et culturelle, organisée par 
l'UNESCO en 2010 à Montréal : « Biocultural diversity [i]s the total sum of the world's differences, no matter what their origin. This concept encompasses biological diversity at all its levels and cultural diversity in all its manifestations » (UNESCO 2010b, art. 12). La "biodiversité culturelle ", telle qu'elle se traduit en français, est ici très largement définie comme «la somme totale des différences dans le monde, quelle que soit leur origine ». Il y a véritablement une unité définie par la différence. La séparation des deux domaines de l'analogie est résolument abolie, et avec elle, l'analogie elle-même s'efface pour faire place à une nouvelle catégorie, à un nouveau concept. Ce phénomène est, d'après Perelman et Olbrechts-Tyteca $(1988: 531-532,535)$, un signe de la fécondité et de la réussite heuristique et cognitive de l'analogie, faisant fusionner deux domaines sémantiques différents. Le phénomène du valisage, perceptible dans la version anglaise de biocultural diversity, dont l'adjectif amalgame les adjectifs biological et cultural, constitue pour Bonhomme (1998: 29) «un procédé privilégié pour explorer le langage ", libérant «la créativité sémantique du langage » et permettant l'élaboration d'une langue affranchie des contraintes du code. Il paraît très approprié pour rendre compte d'une réalité complexe telle que la mondialisation: on trouve, par ailleurs, d'autres exemples d'amalgames lexicaux ou de valisages dans le discours sur la diversité culturelle dans la mondialisation: "glocalisation "(Robertson 1991), fusion entre "globalisation» et «localisation», et «diversité durable» (Appadurai 2003), constitué de "diversité culturelle» et "développement durable». Du point de vue rhétorique, la forme surprenante, condensée et ludique de la formule valisée facilite le contact avec le lecteur et la mémorisation du message, et tente de supprimer les velléités de jugement critique. Ceci permet de minimiser les aspects complexes et contradictoires de la réalité contemporaine (Bonhomme 1998:30) et de fondre deux domaines sémantiques différents sans avoir à démontrer pourquoi.

D'après les auteurs de ce document, les diversités biologique et culturelle sont liées à travers les langues, les savoirs locaux et la relation et la codépendance des peuples autochtones à leur environnement (UNESCO 2010b, art. 10). La notion de "biodiversité culturelle» est donc fondée sur "l'approche écosystémique», selon laquelle «les hommes font partie de la biodiversité " (ibid., 2, art. 8, je traduis). La conception des connaissances traditionnelles comme liant l'homme et son environnement apparait déjà en 1992 dans la Convention de Rio sur la biodiversité, qui reconnaît que les « populations et les communautés autochtones et les autres collectivités locales ont un rôle vital à jouer dans la gestion de l'environnement et le développement du fait de leurs connaissances du milieu et de leurs pratiques traditionnelles " (UNESCO 1992, Annexe 1, principe 22). C'est pourquoi le Sommet de Rio est considéré comme ayant « ouvert la voie à une nouvelle pensée sur les êtres humains et leur environnement, en reliant nature et culture, avec une vision du développement durable " (Bandarín 2011 : 3). La même année, l'UNESCO reconnaît la catégorie des " paysages culturels » comme l'une des catégories du patrimoine culturel de l'humanité (ibid.). Le concept de "paysages culturels ", des paysages où "l'interaction humaine avec les systèmes naturels a formé, sur une longue période, un paysage singulier » (2011:5), reconnait le caractère culturel de l'environnement de l'homme, dans la mesure où celui-ci est inévitablement façonné culturellement par l'homme et investi de valeurs et de sens (17).

37 La citation suivante du document de travail de la Convention pour la biodiversité culturelle illustre la fusion entre les deux types de diversités : 
$2010^{7}$ peut ainsi être considérée comme l'Année célébrant la diversité de la vie sur Terre sous toutes ses formes, mais elle sonne aussi le signal d'alarme sur les changements sans précédent subis par les principales composantes biologiques et culturelles de la planète. Passant des gènes, espèces, écosystèmes, paysages terrestres et marins, aux langues, coutumes, traditions, expressions artistiques et systèmes de croyances, de valeurs et de connaissances, ces diversités font face à des changements sans précédent, allant jusqu'à leur perte. L'impact de la réduction de la diversité bioculturelle sur la résilience des systèmes planétaires est profond. Dans ce contexte actuel de changement mondial, la perte de diversité biologique, jumelée avec la perte simultanée des langues, des systèmes de connaissances et des modes de vie spécifiques, a engendré de nouveaux défis pour les systèmes sociauxécologiques (UNESCO 2010b, je traduis).

La «diversité de la vie sur Terre sous toutes ses formes" réfère évidemment à la biodiversité culturelle définie dans le même document. La diversité culturelle est donc incorporée à " la diversité de la vie sur Terre ", soit à la biodiversité (dont 2010 est l'Année officielle). Le signal d'alarme sonne à la fois pour les composantes biologiques et culturelles de la planète, qui sont incluses en bloc: "Passant des gènes, espèces, écosystèmes, paysages terrestres et marins, aux langues, coutumes, traditions, expressions artistiques et systèmes de croyances, de valeurs et de connaissances». Celles-ci, composantes biologiques et culturelles, appartiennent toutes à la même catégorie de "ces diversités». Elles sont réunies ici en vertu de la menace de disparition pesant sur elles en raison du " contexte actuel de changement mondial ", soit la mondialisation. Les expressions "diversité bioculturelle » et "systèmes socioécologiques » attestent encore de la fusion entre le biologique et le culturel, le social et l'écologique, respectivement. La perception des dangers écologiques est elle aussi perçue «en bloc »: la perte de la diversité biologique est "jumelée avec la perte simultanée des langues, des systèmes de connaissances et des modes de vie spécifiques ».

\section{Conclusion}

On a pu reconstruire une évolution du rapprochement analogique effectué dans le discours de l'UNESCO entre les diversités biologique et culturelle. Cette évolution n'est pas toujours linéaire : on a vu les traces du mélange d'isotopies dans un texte datant de 2000 et la conception écosystémique de l'homme apparaissant déjà en 1992. Le discours social ne répond pas toujours aux schématisations formulées par les rhétoriciens et les analystes du discours : il s'agit d'un objet de recherche complexe, immense, sur lequel nous n'avons pu poser ici qu'un regard microscopique. En outre, les lignes de démarcation comme la transition entre les différents types d'analogies allant jusqu'à leur dépassement sous la forme du mot-valise sont floues et mouvantes. Ceci peut s'expliquer par le statut très flou de la relation entre l'Homme et la Nature, qui fait l'objet de débats: elle va de la séparation totale à l'intégration partielle et à l'assimilation totale entre les deux ${ }^{8}$.

L'argument par analogie a ici de nombreux avantages : outre le fait de transférer à la diversité culturelle une légitimité et un statut déjà établis dans la doxa, le domaine de la biodiversité est également chargé d'une valeur axiologique positive, valeur qu'il transmet au domaine-cible de la diversité culturelle par le moyen de l'analogie. On a également pu repérer, à maintes reprises, un langage provenant d'un registre scientifique ou parascientifique, doublé par le recours à des experts reconnus et 
respectés. Or, malgré ses apparences scientifiques, on a vu comment l'analogie avec la biodiversité pouvait être mobilisée dans un discours idéologique opposé au mouvement de la globalisation. On a pu remarquer que l'association entre diversité biologique et culturelle incluait, généralement, les cultures autochtones, ce qui n'est pas sans faire problème du point de vue éthique. Ce rapprochement analogique parait alors impliquer que seules les cultures autochtones seraient proches de la catégorie Nature, formant un lien symbiotique avec leur environnement.

41 Si l'argument par analogie représente de nombreux avantages, il ne constitue cependant pas un moyen formel de preuve. On peut ainsi se demander, dans le cas de l'analogie entre biodiversité et diversité culturelle, si les deux types de diversité procèdent réellement des mêmes mécanismes d'évolution et si la diversité culturelle est réellement indispensable à la survie de l'espèce Homo sapiens. Ainsi l'article « cultural diversity » du Wikipedia anglais soulève l'objection suivante : " the importance of cultural diversity for survival may be an untestable hypothesis, which can neither be proved nor disproved ». Or, d'après Plantin (2011: 112), c'est justement dans le rapprochement entre des catégories sans possibilités de justifications logiques que réside la force argumentative de l'analogie :

La connaissance analogique est un mode de pensée spécifique, qui s'oppose à la connaissance par les causes, auxquelles sont substituées de mystérieuses correspondances véhiculant des influences. Elle court-circuite la réflexion sur la hiérarchie des catégories en genres et en espèces, à laquelle est substitue une ligne ou un réseau de ressemblances.

En d'autres termes, la démonstration par analogie est une façon de faire connaître le monde là où une démonstration logique est impossible : on est bien dans le domaine de la rhétorique (Perelman et Olbrechts-Tyteca 1988 : 1-2).

\section{BIBLIOGRAPHIE}

Angenot, Marc. 2013. Rhétorique de la confiance et de l'autorité, Discours Social 44

Appadurai, Arjun. 2002. "Diversité durable : l'indivisibilité de la culture et du développement », UNESCO (éd.) Déclaration universelle sur la diversité culturelle. Une vision, une plateforme conceptuelle, une boîte à idées, un nouveau paradigme [En ligne], document établi pour le sommet mondial sur le développement durable, Johannesburg, 26 août - 4 septembre 2002, série « diversité culturelle » 1, Stenou, K. (éd.) (Paris : UNESCO), 9-16, http://unesdoc.UNESCO.org/images/ 0012/001271/127162f.pdf, consulté le 11 décembre 2011

Arizpe, Lourdes \& Ann-Belinda Preis. 2000. « Introduction générale », UNESCO (éd.) Rapport Mondial sur la Culture. Diversité culturelle, conflit et pluralisme [En ligne] (Paris : UNESCO), 14-19, http://unesdoc.UNESCO.org/images/0012/001224/122411F.pdf, consulté le 19 juillet 2015

Arizpe, Lourdes et al. 2000. "Diversité culturelle, conflit et pluralisme », UNESCO (éd.) Rapport Mondial sur la Culture. Diversité culturelle, conflit et pluralisme [En ligne] (Paris : UNESCO), 24-34, http://unesdoc.UNESCO.org/images/0012/001224/122411F.pdf, consulté le 19 juillet 2015 
Bandarín, Francesco. 2011 [2009]. « Préface », Cahiers 26 du Patrimoine mondial, Paysages Culturels du patrimoine mondial. Guide pratique de conservation et de gestion [En ligne] (Paris : UNESCO), 3-4, http://whc.UNESCO.org/fr/series/26/ consulté le 19 janvier 2016

Bonhomme, Marc. 1998. Les figures clés du discours (Paris : Seuil)

Cussó, Roser \& Corinne Gobin. 2008. « Du discours politique au discours expert : le changement politique mis hors débat?», Mots 88, 5-11

Delporte, Christian et al. (éds). 2010. Dictionnaire d'histoire culturelle de la France contemporaine (Paris : Quadrige/PUF)

Garzon, M. (éd.). 2002. Déclaration universelle de l'UNESCO pour la diversité culturelle, Diversité culturelle: Patrimoine commun, identités plurielles [En ligne] (Paris : UNESCO), http:// unesdoc.UNESCO.org/images/0012/001271/127161f.pdf, consulté le 24 février 2011

Hilgert, Emilia. 2016. «L'analogie est-elle plus explicite que la métaphore ? », Langue française 189, 67-86

Koren, Roselyne. 2016. « Exemple historique, comparaison, analogie, métaphore : sont-ils interchangeables? ", Argumentation et Analyse du Discours [En ligne] 16, http://aad.revues.org/ 2123, consulté le 10 avril 2016

Lévi-Strauss, Claude. 2007. « Réflexions », UNESCO. 60 ans de l'histoire de l'UNESCO [En ligne]. Actes du colloque international, Paris, 16 -18 novembre 2005 (Paris : UNESCO), 30- 35, http:// unesdoc.UNESCO.org/images/0015/001541/154122f.pdf, consulté le 15 novembre 2016

Mattelart, Armand. 2007. Diversité culturelle et Mondialisation (Paris : La Découverte)

Organisation des Nations Unies (éd.). 1992. Convention sur la diversité biologique [En ligne], https:// www.cbd.int/doc/legal/cbd-fr.pdf, consulté le 20 mars 2016

Perelman, Chaïm \& Lucie Olbrechts-Tyteca. 1988 [1957]. Traité de l'Argumentation : La Nouvelle Rhétorique (Bruxelles : Éditions de l'Université de Bruxelles)

Plantin, Christian. 2011. « Analogie et métaphore argumentative », A Contrario 16, 110-130

Plantin, Christian. 2016. Dictionnaire de l'argumentation : une introduction aux études d'argumentation (Lyon : ENS Éditions)

Rivière, Françoise. 2010. «Introduction », UNESCO. Catalogue du Festival International de la diversité culturelle 2010 [En ligne] (Paris : UNESCO), http://www.UNESCO.org/fileadmin/MULTIMEDIA/HQ/ CLT/dialogue/pdf/CATALOGUE_ok\%20\%2019-05_01.pdf, consulté le 4 février 2013

Robertson, Roland. 1995. « Glocalization: time-space and homogeneity-heterogeneity », Featherstone, M., S. Lash \& R. Robertson (eds.). Global Modernities (London: Thousand Oaks/New Delhi: Sage, 25-44

Simon, Laurent. 2006. « De la biodiversité à la diversité : les biodiversités au regard des territoires ", Annales de Géographie [En ligne] 651, 2006-5, 451-467, http://www.cairn.info/revueannales-de-geographie-2006-5-page-451.htm, consulté le 20 janvier 2015

UNESCO. 2000. Rapport Mondial sur la Culture 2000. Diversité culturelle, conflit et pluralisme [En ligne] (Paris : UNESCO), http://unesdoc.UNESCO.org/images/0012/001224/122411F.pdf, consulté le 19 juillet 2015

UNESCO. 2005. Convention sur la protection et la promotion de la diversité des expressions culturelles (Paris : UNESCO) [En ligne], http://unesdoc.UNESCO.org/images/0014/001429/142919f.pdf, consulté le 25 novembre 2012 
UNESCO. 2010a. Rapport Mondial de l'UNESCO: Investir dans la diversité culturelle et le dialogue interculturel [En ligne] (Paris : UNESCO), http://unesdoc.UNESCO.org/images/ 0018/001878/187827f.pdf, consulté le 11 décembre 2011

UNESCO. 2010b. Diversity for Development-Development for Diversity [En ligne], International Conference on Biological and Cultural Diversity, June 8-10-2010, Montreal (Working Document), http://www.UNESCO.org/mab/doc/iyb/icbcd_working_doc.pdf, consulté le 26 novembre 2012

UNESCO. 2010c. Catalogue du Festival International de la diversité culturelle 2010 [en ligne] (Paris : UNESCO), http://www.UNESCO.org/fileadmin/MULTIMEDIA/HQ/CLT/dialogue/pdf/ CATALOGUE_ok\%20\%2019-05_01.pdf, consulté le 4 février 2013

Wikipedia. «Cultural diversity » [En ligne], https://en.wikipedia.org/wiki/Cultural_diversity, consulté le 8 janvier 2013

\section{NOTES}

1. Hervé Le Guyader (2008) montre comment, en raison de son flou définitoire et de l'impossibilité de la mesurer, la biodiversité devrait être considérée comme un concept politique et non pas strictement scientifique.

2. Ce travail est basé en partie sur ma thèse de doctorat en cours à l'Université Bar-Ilan, au sujet de « la diversité culturelle dans tous ses états : analyse discursive, rhétorique et argumentative d'une formule».

3. Voir l'article «Écologie, environnement» du Dictionnaire d'histoire culturelle de la France contemporaine (Delporte et al. 2010).

4. Nous développons la catégorie des analogies relationnelles plus loin.

5. Le sociologue Armand Mattelart remarque la même chose quand il dit que diversité culturelle et biodiversité sont des «réalités toutes deux menacées par les logiques prédatrices et inégalitaires du modèle de croissance occidental mû par la consommation excessive des ressources naturelles comme des biens matériels » (2007:97).

6. Delporte et al. $2010: 259-262$.

7. Déclarée par l'UNESCO Année internationale de la diversité biologique.

8. Notamment d'après les tenants de la deep ecology (Delporte et al. 2010, «écologie, environnement »).

\section{RÉSUMÉS}

Nous retraçons les diverses étapes de l'analogie effectuée dans le discours de l'UNESCO entre biodiversité et diversité culturelle, partant de l'article premier de la Déclaration universelle de la diversité culturelle, qui déclare que « la diversité culturelle est, pour le genre humain, aussi nécessaire qu'est la biodiversité dans l'ordre du vivant » (UNESCO 2002). Suivant la typologie de Plantin (2011) - analogie relationnelle, catégorielle et structurelle -, nous reconstruisons, à l'aide d'analyses détaillées de textes publiés par l' UNESCO entre 2001 et 2010, la trajectoire de cette analogie, qui évolue à travers ces trois stades jusqu'à la fusion des deux notions en celle de la «biodiversité culturelle» («biocultural diversity») (UNESCO 2010b, art. 12). Nous nous arrêtons 
sur la valeur argumentative des analogies analysées, qui inclut la légitimation de la diversité culturelle à travers un concept-valeur déjà reconnu et stabilisé dans la doxa (la biodiversité), et la naturalisation d'un concept politique par son association de plus en plus étroite à un concept scientifique et «naturel ». Cet article se penche également, au passage, sur le rôle argumentatif du flou catégoriel dans le langage.

We trace the different stages of the analogy found in UNESCO's discourse between biological and cultural diversity. The first article of the Universal Declaration on Cultural Diversity, stating that "cultural diversity is as necessary to humankind as biodiversity is to nature" (UNESCO 2002) serves as our starting point. Following Plantin's typology of analogies (2011): relational, categorial and structural, we analyze various UNESCO documents between 2001 and 2010 in order to reconstruct the trajectory of this analogy as it evolves through those three different stages, until it merges into "biocultural diversity", a concept formulated in a 2010 UNESCO conference (UNESCO 2010b, art. 12). We furthermore underline the argumentative value of this analogy, which includes the legitimization of cultural diversity through a concept and value already widely accepted in the doxa (biodiversity) as well as the naturalization of a political concept cultural diversity - through its close association with a scientific and "natural" one. The article also explores the argumentative role of fuzzy semantic and categorial boundaries.

\section{INDEX}

Mots-clés : analogie, biodiversité, diversité culturelle, rhétorique argumentative, UNESCO

Keywords : analogy, biodiversity, cultural diversity, rhetoric argumentation, UNESCO

\section{AUTEUR}

\section{IRIT SHOLOMON-KORNBLIT}

Université Bar-Ilan, ADARR 